\title{
Estimación de la Capacidad de una Institución de Educación Superior Usando Dinámica de Sistemas: Caso de Estudio Escuela Colombiana de Ingeniería Julio Garavito
}

\section{Capacity Estimation in a Higher Education Institution Using System Dynamics: Case of Study Escuela Colombiana de Ingeniería Julio Garavito}

\author{
Carlos Rodrigo Ruiz-Cruz, MSc${ }^{1}$, Sonia Alexandra Jaimes Suarez, MSc ${ }^{1}$ \\ ${ }^{1}$ Escuela Colombiana de Ingeniería Julio Garavito, Colombia, carlosr.ruiz@escuelaing.edu.co, sonia.jaimes@escuelaing.edu.co
}

\begin{abstract}
Resumen-Mejorar la calidad de la educación es el objetivo más importante para cualquier institución educativa. Para el logro del mismo se requiere de una adecuada gestión de los recursos necesarios para el desarrollo del quehacer académico. El propósito de este proyecto es estimar la capacidad de una institución de educación superior, en particular para los programas de pregrado de la Escuela Colombiana de Ingeniería Julio Garavito. Se utiliza dinámica de sistemas como herramienta para lograr este objetivo. El modelo permite estimar el efecto de la admisión en el horizonte definido sobre recursos como salones, laboratorios y profesores. Para el desarrollo y evaluación del modelo se utilizan datos históricos desde el año 2000 y se prueban diferentes escenarios de admisión.

Palabras claves: Capacidad, dinámica de sistemas, gestión académica.
\end{abstract}

Abstract- The education quality improvement is the main objective for any educational institution. To achieve such purpose, it requires proper resource management of those needed to accomplish the academic processes. In this proyect the capacity of a higher education institution is estimated, in particular the undergraduate programs at Escuela Colombiana de Ingenieria Julio Garavito. System dynamics was used to achieve this objective. The model allows to estimate the effect in the horizon of admissions over resources like classrooms, laboratories and teachers. In order to develop and evaluate this model, historical data since 2000 and different scenarios of admission are tested.

Keywords-- Capacity, system dynamics, academic management.

\section{INTRODUCCIÓN}

La Escuela Colombiana de Ingeniería Julio Garavito fue fundada en el año 1972, y desde entonces ha venido ofreciendo una amplia oferta académica. Actualmente cuenta con 11 programas de pregrado, 9 especializaciones y 6 maestrías. La institución es reconocida por su excelencia académica y se encuentra dentro de las instituciones de educación superior en
Colombia que cuentan con Acreditación Institucional de Alta Calidad.

Las políticas de admisión de la Institución no tienen definido un máximo de aspirantes que puedan ser admitidos a sus programas académicos. Sin embargo, es deseable saber previamente la cantidad máxima de estudiantes que pueden ingresar a cada programa de pregrado y cómo es su comportamiento a través del tiempo, debido al efecto sobre los recursos disponibles que genera el flujo de estudiantes, buscando siempre mantener la máxima calidad educativa. La institución realizó un proceso de renovación curricular que implicó la actualización de los planes de estudios de todos sus programas de pregrado lo que generó incertidumbre sobre los efectos que estos cambios podrían generar sobre la capacidad de la institución y de esta necesidad surge este proyecto. En el año 2015 se desarrolló una primera aproximación a resolver esta problemática en particular en el Programa de Ingeniería Industrial [1]. Los resultados permitieron identificar la capacidad máxima del programa con los recursos propios con los que disponía en el momento, sin embargo, se identificó que debido a que muchos recursos se compartían con otros programas y departamentos, para llegar a conclusiones más relevantes se debía considerar a toda la institución como un solo sistema cuyos componentes eran sus programas académicos y que se interrelacionaban de manera permanente a través del tiempo. De tal forma nació este proyecto tratado de dar respuesta a esta problemática.

La capacidad se define como el potencial que tiene un sistema para llevar a cabo las funciones para las cuales fue diseñado [2], en el caso de los sistemas de servicios la estimación de la misma representa un problema de difícil manejo debido a su naturaleza, diversidad y complejidad. Trabajos orientados a este objetivo se han desarrollado en 
servicios turísticos. Como el caso del Parque Nacional Galápagos en Ecuador [3] para el cual se estableció la carga máxima, en número de visitantes admitidos a las diferentes áreas del parque. Ese estudio consideró el impacto que tienen los visitantes sobre el entorno. Serra [4] propuso un marco integrado para el estudio de las dinámicas en sistemas turísticos. El modelo desarrollado por este autor considera que la capacidad se ve afectada por una interacción dinámica entre los elementos estructurales y los agentes participantes en las diversas dimensiones del sistema turístico. En este proyecto se modela el comportamiento de los programas académicos de pregrado usando dinámica de sistemas.

\section{MetodologíA}

Para el desarrollo del modelo se realizaron las siguientes etapas:
A. Identificación de parámetros.
B. Recolección de la información.
C. Análisis y tratamiento de los datos.
D. Construcción del modelo de dinámica de sistemas.
E. Validación de resultados con los programas académicos.
F. Desarrollo de escenarios.

A continuación, se detallan cada una de las etapas:

\section{A. Identificación de parámetros relevantes del sistema}

$\mathrm{Al}$ analizar las estructuras de los planes de estudios y las políticas establecidas por la institución, se identificaron en cada uno de los programas de pregrado los siguientes parámetros:

- Estructura del plan de estudios.

- Requerimientos de las asignaturas del plan de estudios: - Número de sesiones a la semana de cada asignatura. - Número de estudiantes por grupo en cada asignatura. - Laboratorios utilizados pos la asignatura.

- Número de profesores de planta disponibles para orientar cada asignatura.

- Porcentaje de estudiantes que aprueban, reprueban o cancelan cada asignatura por periodo.

- Número de estudiantes inscritos, admitidos y matriculados al programa.

- Deserción estudiantil del programa.

- Clasificación de ingreso en las áreas de matemáticas, física, química e idiomas.

- Perfiles de los profesores de planta de los programas.

\section{B. Recolección de la información}

Los datos utilizados se obtuvieron del base de datos de la Oficina de Registro y Control Académico, Oficina de Admisiones, Departamentos, Unidades y Decanaturas. Se utilizó información desde el año 2000 hasta 2017. Este intervalo de tiempo garantiza la inclusión de observaciones de varias cohortes de los programas.

\section{Análisis y tratamiento de los datos}

La variable que afecta directamente la capacidad de atención es la admisión semestral de estudiantes nuevos (demanda), quienes cursan el plan de estudios a medida que aprueban las asignaturas que lo componen. A partir de la información histórica del comportamiento de esta variable se realizaron pronósticos de su posible comportamiento futuro y escenarios para considerar como tendencias diferentes a la actual podían afectar la capacidad de la institución.

\section{Construcción del modelo de dinámica de sistemas}

Con el fin de analizar la interacción que tienen las diferentes variables a través del tiempo, se utilizó como herramienta Dinámica de Sistemas (DS), la cual es una metodología para el análisis y simulación de sistemas complejo [5]. Para el desarrollo del modelo se establecieron los siguientes supuestos:

- Los planes de estudios tienen asignaturas comunes coordinadas por los Departamentos o Unidades, asignaturas propias de cada programa y asignaturas que los programas comparten con otros.

- Se aplican tasas de absorción que consideran el paso de los aspirantes por los estados de inscrito, admitido y matriculado. Estas fueron calculadas a partir de la información histórica de cada programa.

- Los estudiantes se rigen al orden establecido en el plan de estudio de su programa para cursar las asignaturas.

- Existen requisitos para cursar las asignaturas. Estos se definen como las asignaturas que deben ser cursadas y aprobadas previamente.

- En caso de que una asignatura tenga más de un prerrequisito, se toma aquel cuyo flujo de estudiantes sea menor.

- Las electivas de los programas y departamentos no se consideran en de forma detallada sino como una única asignatura ofertada.

- Se aplican una tasa de aprobación a cada asignatura, calculadas a partir de la información histórica de cada asignatura. Esta se define como el porcentaje de alumnos que aprueban una asignatura.

- Las tasas de aprobación son calculadas con el promedio ponderado basado en el número de estudiantes de cada programa que inician cursando las asignaturas, en algunos casos, por decisión del programa, este valor es reemplazado por el promedio de los últimos dos años.

- Se aplica una tasa de permanencia que se define como el porcentaje de alumnos que continúan en el programa entre un semestre académico y otro, y es aplicada al flujo de salida de cada una de las asignaturas, es decir, a los estudiantes que la aprueban. Esta fue calculada a partir de la información histórica de cada programa.

- Se aplica una tasa de cancelación a cada asignatura, calculada a partir de la información histórica. Esta se define como el porcentaje de alumnos que cancelan una asignatura que inicialmente inscribieron en el periodo académico.

Digital Object Identifier: (to be inserted by LACCEI).

ISSN, ISBN: (to be inserted by LACCEI).

16 $^{\text {th }}$ LACCEI International Multi-Conference for Engineering, Education, and Technology: "Innovation in Education and Inclusion", 19-21 July 2018, Lima, Peru. 
- Cada programa determinó una asignatura habilitadora, que funciona como un prerrequisito para las asignaturas electivas y las opciones de grado.

De acuerdo a lo anterior, se desarrolló el Diagrama de Forrester [5] que representa la situación estudiada y permite establecer las ecuaciones matemáticas que definen el comportamiento del sistema. En el Diagrama de Forrester se consideran los siguientes elementos:

- Niveles: variables que acumulan, o consumen, recursos. En el modelo desarrollado cada asignatura tiene un nivel y representa el número de estudiantes que la están cursando. Así mismo, el número de estudiantes admitidos se representan con un nivel por cada programa académico.

- Flujos: variables que representan la tasa de cambio de un nivel. En el modelo representan el flujo de estudiantes que se matriculan, así como los que ingresan a una asignatura, los que la aprueban, y los que reprueban o cancelan.

- Convertidores o variables auxiliares: variables o constantes que toman datos de entrada y los convierten en una señal de salida. En el modelo representan las tasas de aprobación, permanencia, clasificación inicial en matemáticas y ciencias naturales, porcentaje de admitidos y porcentaje de matriculados.

- Conectores: indican las relaciones causales entre elementos del modelo. En el modelo representan las relaciones de impacto de la aprobación, permanencia, conversión del flujo de salida de estudiantes aprobados en el flujo de entrada para otras asignaturas, los estudiantes reprobados o cancelados y los requisitos de las asignaturas. Así mismo, las relaciones entre inscritos, admitidos y matriculados.

Se utilizó el software Ithink® para representar gráficamente el modelo, conceptualizar, documentar, simular y analizar las interacciones de los elementos del sistema. En la Fig. 1 se muestra la estructura del modelo para la admisión de estudiantes. La entrada al sistema se da por medio del flujo "Admitidos programa" el cual resulta de multiplicar las variables "Inscritos programa" y "Porcentaje de admitidos del programa", el nivel representa los alumnos nuevos que cumplen con los criterios de admisión definidos por la institución, los cuales pueden tomar dos decisiones, la primera es matricularse y con lo cual se convierten en el flujo "Matriculados programa" el cual es el resultado del nivel "Alumnos nuevos programa" multiplicado por la variable "Porcentaje de matriculados del programa". La segunda decisión puede ser no matricularse con lo cual se convierten en el flujo "No matriculados programa" el cual es calculado al multiplicar el nivel "Alumnos nuevos programa" por 1 menos el "Porcentaje de matriculados del programa".

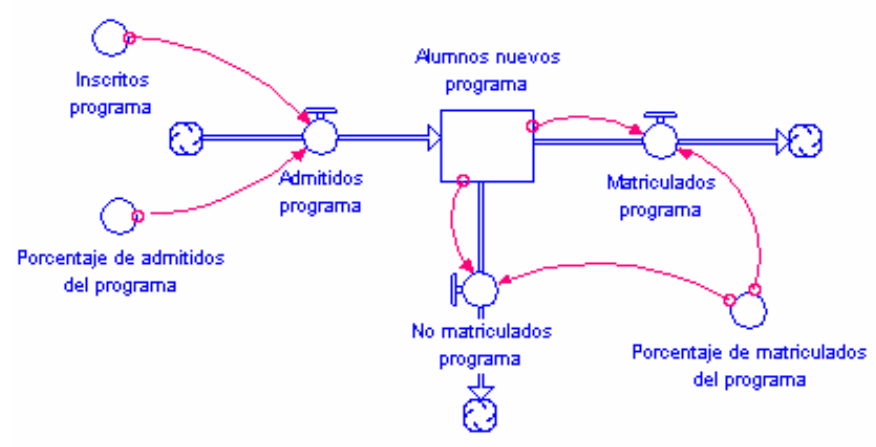

Fig. 1 Modelo de admisión de estudiantes a los programas

En la Fig. 2 se muestra la estructura del modelo de comportamiento de una asignatura. El nivel representa el número de "Estudiantes cursando la asignatura" el cual aumenta o disminuye de acuerdo a la entrada de estudiantes con el flujo de entrada "Estudiantes que ingresan a la asignatura" y disminuye con los flujos de salida "Estudiantes que aprueban la asignatura" y con el flujo "Estudiantes que pierden o cancelan la asignatura". Para el cálculo del flujo de entrada se toman como referencia las variables "Asignatura requisito" que corresponde al flujo de salida ("Estudiantes que aprueban la asignatura") de aquellas asignaturas que deben ser cursadas previamente. En caso de ser solo una se toma el flujo igual a la variable, en caso de ser más de un requisito se toma el valor de la asignatura que tenga el menor flujo, a lo anterior se aplican retrasos para las asignaturas que no son cursadas en el periodo académico inmediatamente anterior y se afecta por la tasa de "Permanencia del semestre $j$ al semestre $j$ más 1" que corresponde al porcentaje de estudiantes en el semestre $j$ del plan de estudios menos el porcentaje de deserción histórico del semestre $j$ al $j+1$ del plan de estudios. Para el cálculo del flujo de salida "Estudiantes que aprueban la asignatura" se multiplica el nivel por la variable "Aprob. de la asignatura", que corresponde al porcentaje histórico de estudiantes que la aprueban. El flujo "Estudiantes que pierden o cancelan la asignatura" corresponde al nivel multiplicado por 1 menos "Aprob. de la asignatura". 


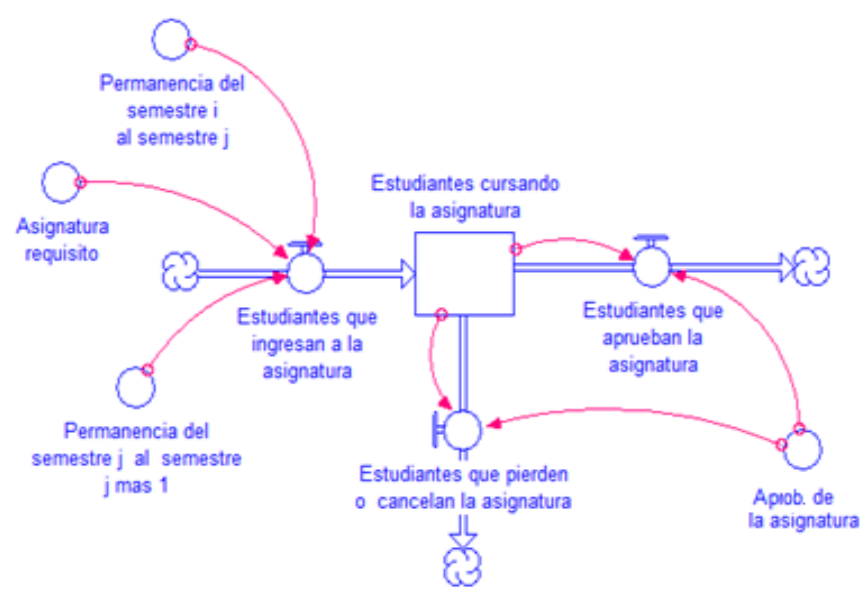

Fig. 2 Modelo de comportamiento de las asignaturas
En la Fig. 3 se muestra de manera general parte de la estructura del modelo. En la zona 1 se modela el comportamiento de las admisiones a cada uno de los programas considerados (cada color corresponde al flujo de un programa académico de pregrado). En la zona 2 se modelan las asignaturas del núcleo común, que corresponden a aquellas que los estudiantes deben cursar en su formación sin importar el programa académico. En estas se consideran la formación en matemáticas, ciencias naturales y humanidades. En la zona 3 se modelan las asignaturas compartidas, que son aquellas ofrecidas, y administradas académicamente, por un programa académico, pero que estudiantes de otros programas deben cursar como parte de su plan de estudios. Por último, en la zona 4 se modela el comportamiento de los planes de estudios de cada programa académico y sus asignaturas propias.

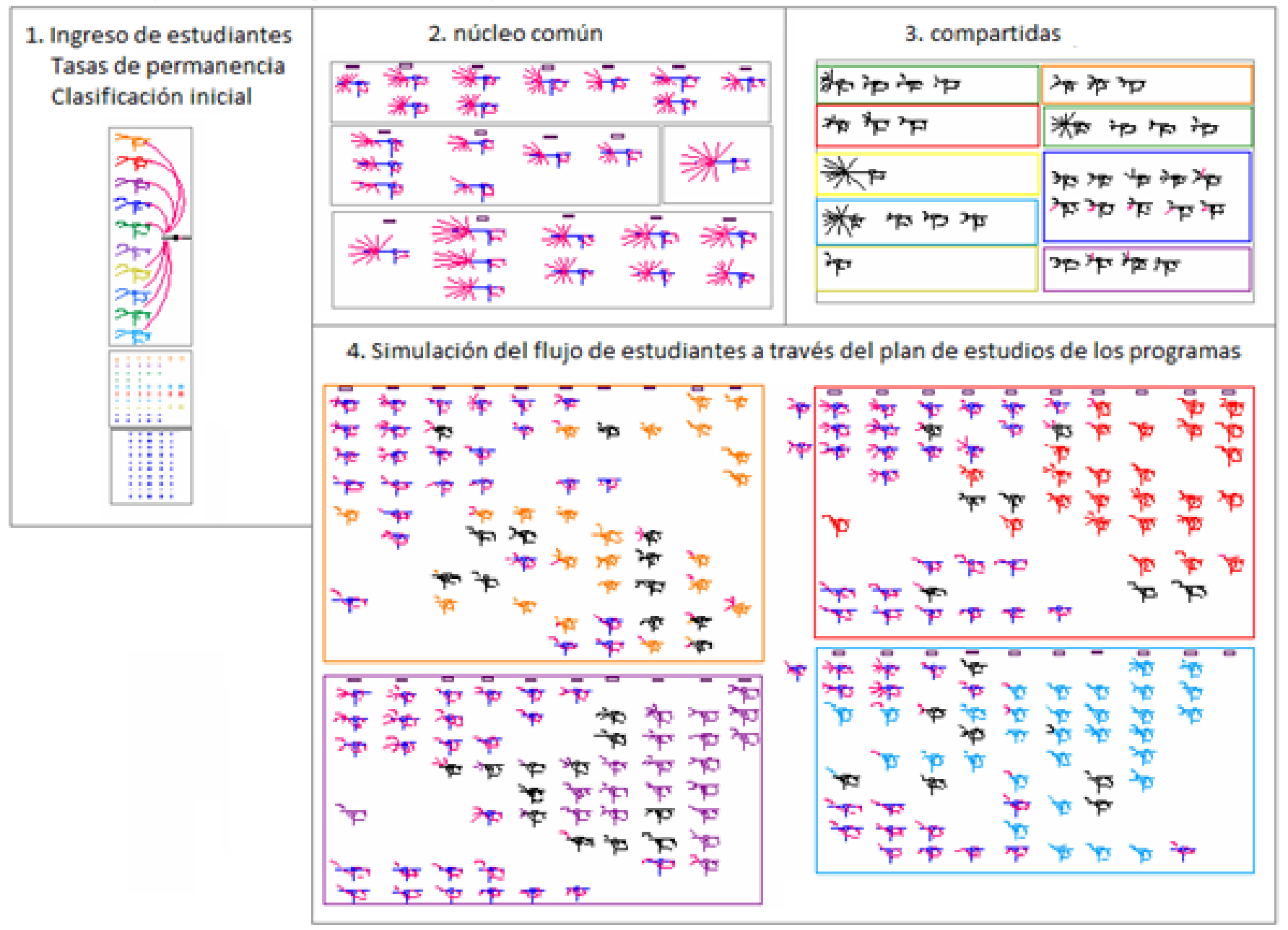

Fig. 3 Modelo de comportamiento de las asignaturas

E. Validación de resultados preliminares con los programas académicos

Se realizó una validación cuantitativa y cualitativa con los programas y departamentos en la cual se buscó establecer el comportamiento de los resultados del modelo en comparación a los datos históricos. Cuantitativamente se realizó un análisis de correlación encontrando en general altos valores del coeficiente de correlación. De forma cualitativa cuando se

16 $^{\text {th }}$ LACCEI International Multi-Conference for Engineering, Education, and Technology: "Innovation in Education and Inclusion", 19-21 July 2018, Lima, Peru. 
encontraron brechas significativas se identificaron causas como cambios en el plan de estudios (ubicación de las asignaturas dentro del plan, cambios en los requisitos y eliminación de correquisitos), el supuesto de que el estudiante cursa las asignaturas en el semestre en que propone el plan de estudios, efectos del periodo intersemestral, ofrecimiento o eliminación de la asignatura en otros programas académicos, asignaturas validadas como electivas técnicas en otros programas y el efecto de estudiantes de doble programa y transferencias. Finalmente se concluyó que el modelo permitía representar de forma fiable el comportamiento de los programas académicos y sus asignaturas.

\section{F. Desarrollo de escenarios}

Con el fin de evaluar el efecto que pueden generar cambios en comportamiento de los aspirantes a los programas de pregrado de la institución y su impacto sobre los recursos necesarios se desarrollaron tres escenarios base:

1. Escenario constante. En este escenario se realiza la simulación suponiendo que durante los próximos 5 años las admisiones fueran el promedio de los últimos 5 años (Fig. 4).

2. Escenario optimista. En este escenario se realiza la simulación suponiendo que durante los próximos 5 años las admisiones mantienen la tasa de crecimiento que se ha observado en los últimos 5 años (Fig. 5).

3. Escenario pesimista. En este escenario se realiza la simulación suponiendo que durante los próximos 5 años las admisiones se decrecen a una tasa entre el $3 \%$ y el $6 \%$ (dependiendo del programa) a partir de los últimos periodos académicos conocidos (Fig. 6).

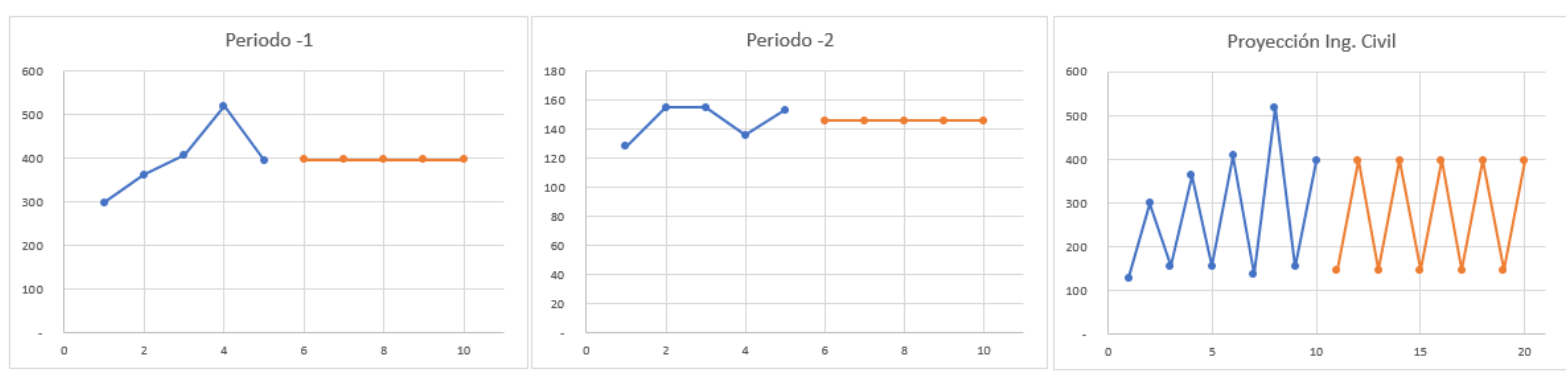

Fig. 4 Proyecciones de admisión al Programa de Ingeniería Civil en el escenario CONSTANTE

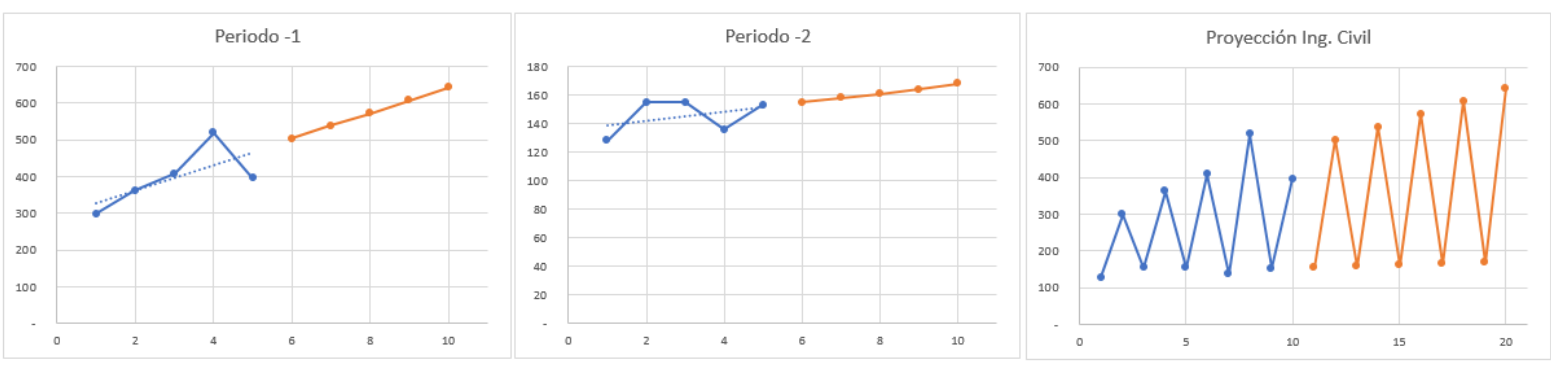

Fig. 5 Proyecciones de admisión al Programa de Ingeniería Civil en el escenario OPTIMISTA
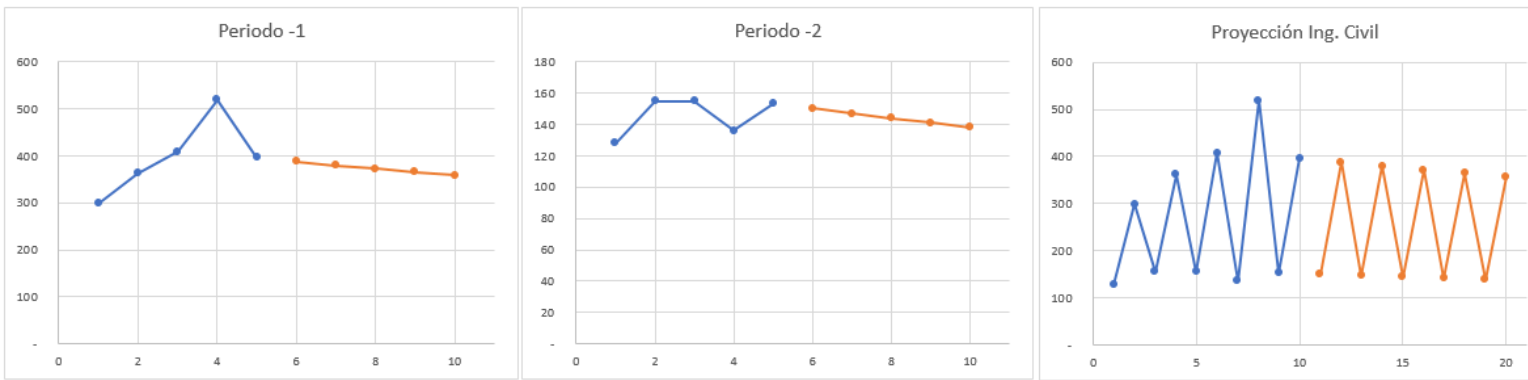

Fig. 6 Proyecciones de admisión al Programa de Ingeniería Civil en el escenario PESIMISTA

\section{RESULTADOS}

Los resultados de la simulación generan una estimación del número de estudiantes inscritos por periodo en cada una de las asignaturas que se ofrecen en la institución. Esta variable se denota como $N E_{i}$. En la Tabla I se presenta a manera de ejemplo parte de los resultados de la simulación del escenario CONSTANTE en las asignaturas correspondientes a la formación en el área matemática: Precálculo (PREM), Cálculo Diferencial (CALD), Cálculo Integral (CALI) y Cálculo Vectorial (CALV). 
EJEMPLO DE RESULTADOS DE LA SIMULACIÓN

\begin{tabular}{|c|c|c|c|c|}
\hline Periodo & PREM & CALD & CALI & CALV \\
\hline $2017-2$ & 855 & 906 & 666 & 595 \\
\hline $2018-1$ & 1282 & 997 & 639 & 607 \\
\hline $2018-2$ & 947 & 984 & 677 & 587 \\
\hline $2019-1$ & 1380 & 1081 & 681 & 612 \\
\hline $2019-2$ & 1005 & 1055 & 730 & 618 \\
\hline $2020-1$ & 1462 & 1148 & 731 & 655 \\
\hline $2020-2$ & 1057 & 1117 & 777 & 663 \\
\hline $2021-1$ & 1543 & 1211 & 774 & 700 \\
\hline $2021-2$ & 1110 & 1176 & 820 & 705 \\
\hline $2022-1$ & 1623 & 1276 & 815 & 741 \\
\hline
\end{tabular}

El análisis de los resultados de los escenarios se realiza teniendo en cuenta los siguientes parámetros:

$T G_{i}$ : Tamaño de los grupos de la asignatura $i$. Corresponde al número máximo de estudiantes que pueden constituir un grupo de clase de la asignatura. Este es definido por cada programa académico o departamento y oscila entre 15 y 40 estudiantes.

$T G L_{i}$ : Tamaño de los grupos de laboratorio de la asignatura $i$. Corresponde al número máximo de estudiantes que pueden constituir un grupo de laboratorio de una asignatura. Este es definido por cada programa académico o departamento y oscila entre 5 y 15 estudiantes.

$S_{i}$ : Sesiones de la asignatura $i$ por semana. Corresponde al número de sesiones por semana que se tienen definidas por cada asignatura en función de su número de créditos académicos y presencialidad requerida. Este es definido por cada programa académico o departamento y oscila entre 2 y 4 sesiones por semana.

$S L_{i}$ : Sesiones de laboratorio de la asignatura $i$ por semana. Corresponde al número de sesiones de laboratorio por semana que se tienen definidas por cada asignatura. Este es definido por cada programa académico o departamento oscila entre 1 y 2 sesiones por semana.

$L_{i j}$ : Parámetro que toma el valor de 1 si el laboratorio de la asignatura $i$ requiere para su desarrollo el espacio de laboratorio tipo j. 0 en caso contrario.

$C P$ : Carga horaria semanal profesor de planta. Corresponde al número máximo de horas por semana que un profesor de planta dedica a la docencia en clases presenciales. Por política institucional es 12 para los profesores de departamento y 9 para los profesores de los programas.

$R P C$ : Relación planta-cátedra. Corresponde a la proporción del número de horas de clase necesarias que se asumen por el personal de planta de la institución. Por política institucional se he definido que un tercio de las horas necesarias sean asumidas por la planta y los dos tercios restantes por profesores de cátedra.

$D S C$ : Duración de las sesiones de clase. En la institución las sesiones de clase son de 1.5 horas.

$F$ : Franjas disponibles por salón por semana. Corresponde al número máximo de sesiones de clase por semana que se pueden programar en un salón de clases. En la institución se tiene un horario de trabajo académico de lunes a viernes de 7:00 a 19:00 y sábados de 7:00 a 13:00, y dado que la duración de las sesiones de clase $(D S C)$ es de 1.5 horas. De tal forma el número disponible de franjas por salón a la semana es de 44, sin embargo, en la práctica no todas estas sesiones pueden ser utilizadas en razón a la disponibilidad de los profesores y las preferencias de programación de las asignaturas, entre otros, de tal forma para el proyecto se estimó que el número máximo disponible sería 38.

$F L_{j}$ : Franjas disponibles por laboratorio tipo $j$ por semana. Corresponde al número máximo de sesiones de laboratorio por semana que se pueden programar en el laboratorio j. Así como para los salones el número máximo disponible es 38.

Los indicadores definidos para el análisis de los resultados por periodo fueron:

$G C_{i}$ : Grupos de clase de la asignatura $i$ (1). Número necesario de grupos que se necesitarían para atender al total de estudiantes que cursan la asignatura $i$.

$G C_{i}=\frac{N E_{i}}{T G_{i}}$

GCT: Grupos de clase totales (2). Número total de grupos de clase que se requieren en la institución en un periodo determinado.

$G C T=\sum_{i} G C_{i}$

$H_{i}$ : Horas de clase de la asignatura $i$ (3). Número de horas necesarias por semana por asignatura $i$.

$H_{i}=G C_{i} \times S_{i} \times D S C$

HT: Grupos de clase totales (4). Número total de horas de clase que se requieren en la institución en un periodo determinado.

$H T=\sum_{i} H_{i}$

$S C_{i}$ : Salones de clase necesarios para la asignatura $i$ (5). Número de salones necesarios para orientar las sesiones de clase por semana del total de grupos de cada asignatura $i$.

$S C_{i}=\frac{H_{i}}{F \times D S C}$

SCT: Salones de clase totales (6). Número total de salones de clase que se requieren en la institución en un periodo determinado.

$S C T=\sum_{i} S C_{i}$

$G L_{i}$ : Grupos de laboratorio de la asignatura $i$ (7). Número necesario de grupos de laboratorio que se necesitarían para atender al total de estudiantes que cursan la asignatura $i$.

$G L_{i}=\frac{N E_{i}}{T G L_{i}}$

$G L T_{j}$ : Grupos de laboratorio tipo $j$ totales (8). Número total de grupos de laboratorio que se requieren en la institución en un periodo determinado y que se realizan en el espacio de laboratorio tipo $j$.

$G L T_{j}=\sum_{i} G L_{i} \times L_{i j} \quad \forall j$

16 $^{\text {th }}$ LACCEI International Multi-Conference for Engineering, Education, and Technology: "Innovation in Education and Inclusion", 19-21 July 2018, Lima, Peru. 
$H L_{i}$ : Horas de laboratorio de la asignatura $i$ (9). Número de horas necesarias de laboratorio por semana por asignatura $i$.

$H L_{i}=G L_{i} \times S L_{i} \times D S C$

HT: Grupos de clase totales (10). Número total de horas de clase que se requieren en la institución en un periodo determinado.

$H T=\sum_{i} H_{i}$

$L_{j}$ : Espacios de laboratorio tipo $j$ necesarios (11). Número de espacios de laboratorio del tipo $j$ necesarios para orientar todas las sesiones de laboratorio que requieren su utilización.

$L_{j}=\frac{\sum_{i} G L_{i} \times S L_{i} \times L_{i j}}{F L_{j}} \quad \forall j$

$P P_{i}$ : Profesores de planta para la asignatura $i$ (12). Número necesario de profesores de planta que se necesitarían para atender al total de grupos de la asignatura $i$.

$P P_{i}=\frac{H_{i} \times R P C}{C P}$

PPT: Profesores de planta totales (13). Número necesario de profesores de planta que se necesitarían en la institución. $P P T=\sum_{i} P P_{i}$

$H C_{i}$ : Horas de cátedra para la asignatura $i$ (14). Número necesario de horas cátedra que se necesitarían para atender al total de grupos de la asignatura $i$.

$H C_{i}=H_{i} \times(1-R P C)$

HCT: Horas de cátedra totales (15). Número necesario de horas de cátedra que se necesitarían en la institución.

$H C T=\sum_{i} H C_{i}$

Los resultados permitieron evidenciar la necesidad que tendría la institución de aumentar su capacidad en particular en salones de clase y profesores, ya que inclusive en el escenario PESIMISTA se observa que el número actual sería insuficiente para atender el número de grupos de clase necesarios si se analiza un horizonte de 5 años, tal como se observa en las Fig. 7 y 8 , considerando que en la actualidad la institución cuenta con 73 salones disponibles y 150 profesores de planta. Estos resultados llevaron a que la institución tomara la decisión de construir un nuevo edificio para atender exclusivamente los programas de posgrado y de esta forma liberar uno de los edificios que en este momento cuenta con salones dedicados de uso compartido entre pregrado y posgrado. De igual forma se revisaron decisiones relacionadas con la presencialidad de las asignaturas, la conformación de los grupos de clase y las características de las asignaturas del área de idiomas.

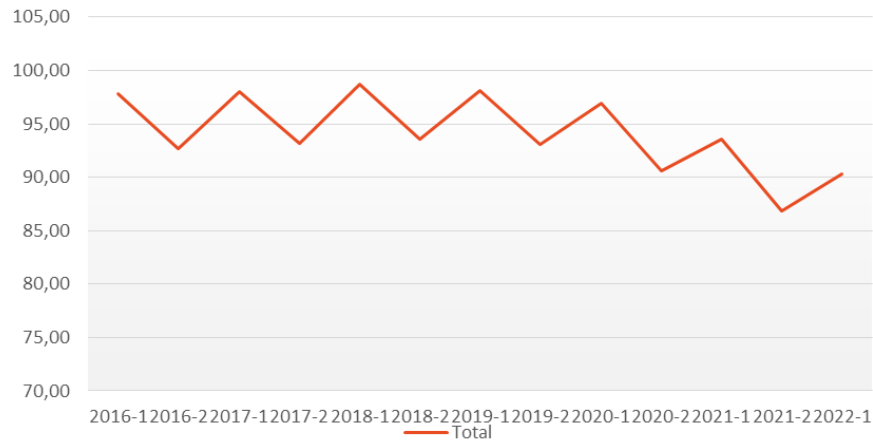

Fig. 7 Salones de clase necesarios escenario PESIMISTA

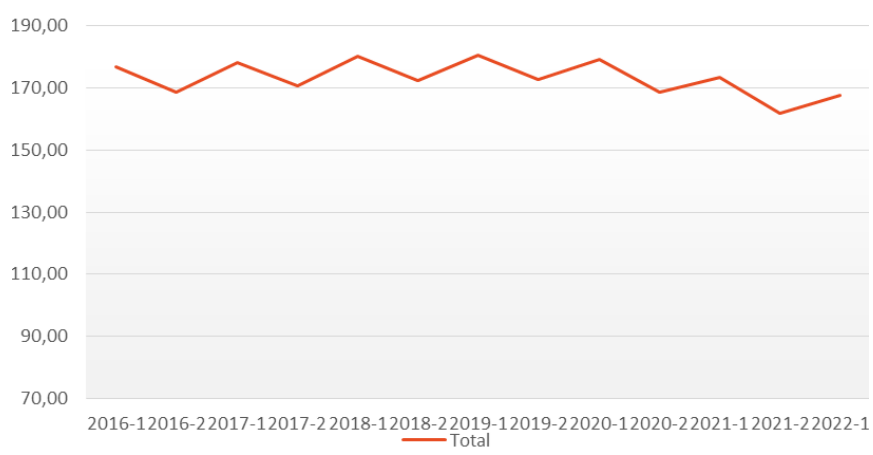

Fig. 8 Profesores de planta necesarios escenario PESIMISTA

En relación a los profesores las simulaciones permiten determinar el perfil o área de actuación de los profesores, por lo cual se puede definir un plan de desarrollo profesoral y vinculación de acuerdo a las áreas que se requerirían cubrir en el futuro.

Por otra parte, contrario a una hipótesis que se manejaba en la institución los espacios de laboratorio no son un cuello de botella en la capacidad, ya que se demostró en las simulaciones de los escenarios base que el uso de la capacidad utilizada estaba entre el $8 \%$ y el $90 \%$ y solamente en el escenario OPTIMISTA uno de los espacios de laboratorio (Laboratorio de Informática del Departamento de Matemáticas) usaba el $100 \%$ de su capacidad. Sin embargo, en el análisis se debió tener en cuenta que los espacios de laboratorio también se utilizan para el desarrollo de prácticas libres por parte de los estudiantes y el desarrollo de proyectos de investigación. De tal forma se identificaron aquellos que por su alta utilización podrían ser susceptibles de ampliaciones o adecuación de nuevos espacios en el mediano plazo.

\section{CONCLUSIONES Y TRABAJO FUTURO}

El modelo propuesto permite hacer una medición de la capacidad necesaria en cualquier institución educativa, considerando las características propias de sus programas académicos ayudando a soportar los procesos de toma de decisiones a mediano y largo plazo, evaluando la capacidad instalada y brindando insumos para las decisiones relacionadas con los procesos y políticas de admisión, la gestión y la planeación de recursos físicos y humanos. En la institución

16 $^{\text {th }}$ LACCEI International Multi-Conference for Engineering, Education, and Technology: "Innovation in Education and Inclusion", 19-21 July 2018, Lima, Peru. 
objeto de estudio el modelo ha permitido soportar decisiones estratégicas respecto a sus recursos, en particular nueva infraestructura física, ya que permite prever con anticipación el impacto de las mismas.

A la fecha se han desarrollado más escenarios, que se espera permitan analizar nuevas políticas de admisión, como por ejemplo la definición de cupos máximos por programa, la modificación de los horarios académicos y estrategias de virtualización del currículo, entre otras.

En la actualidad está en desarrollo el modelo de estimación de la capacidad para los programas de posgrado que permitirá analizar de manera integral la capacidad de la institución. El modelo integral pregrado-posgrado se espera que pueda alimentar un modelo de análisis financiero que permita evaluar en este sentido el impacto de las decisiones académicas sobre las finanzas institucionales. De igual forma se espera poder desarrollar una herramienta que sirva de interfaz entre los potenciales usuarios del modelo (Rector, Vicerrectores, Decanos, Jefes de Departamento) con el fin de que puedan realizar sus propias simulaciones y análisis.

\section{AGRADECIMIENTOS}

Agradecemos a la Escuela Colombiana de Ingeniería Julio Garavito por el apoyo en el desarrollo e implementación de este proyecto, en particular a los rectores Germán Eduardo Acero Riveros y Myriam Astrid Angarita Gómez quienes nos brindaron todo su apoyo, los recursos necesarios y sobre todo su confianza en el potencial que estos resultados pueden generar para el desarrollo de la prospectiva de la institución.

\section{REFERENCIAS}

[1] S. A. Jaimes Suarez, C. R. Ruiz-Cruz, C. Castro, S. Gordillo, S. Meza, and J. Niño, "Estimación de la capacidad del Programa de Ingeniería Industrial de la Escuela Colombiana de Ingeniería Julio Garavito," Rev. la Esc. Colomb. Ing., vol. XXVII, no. 106, pp. 69-76, 2017.

[2] P. H. Pittman and J. B. Atwater, APICS Dictionary, 15 th. Chicago, IL.: APICS, 2016.

[3] P. Amador, E., Cayot, L., Cifuentes, M., Cruz, E., Cruz, F., \& Ayora, "Determinación de la capacidad de carga turística en los sitios de visita del Parque Nacional Galápagos," Serv. Parq. Nac. Galápagos, Ecuador., p. 42, 1996.

[4] F. M. D. Serra, "Modelización del sector turístico y simulación de estrategias mediante dinámica de sistemas: aplicación al Agarbe portugués," Universidad de Huelva, 2003.

[5] J. W. Forrester, "System dynamics, systems thinking, and soft OR," Syst. Dyn. Rev., vol. 10, no. 2-3, pp. 245-256, 1994.

16 $^{\text {th }}$ LACCEI International Multi-Conference for Engineering, Education, and Technology: "Innovation in Education and Inclusion", 19-21 July 2018, Lima, Peru. 